\title{
Concomitant Aspergillus Species Infection and Squamous Cell Carcinoma Diagnosed on Pap Smear
}

\author{
Prajwala GUPTA, Snigdha GOYAL, Manju KAUSHAL \\ Department of Pathology, PGIMER, Dr. RML Hospital, NEW DELHI, INDIA
}

\begin{abstract}
Concomitant infection with Aspergillus species and cervical squamous cell carcinoma in the female genital tract is a rare occurrence and attributed to the opportunistic nature of infection in the immunocompromised state due to the underlying malignancy. The contamination of smears with Aspergillus species should be excluded. The diagnosis of Aspergillus species infection along with squamous cell carcinoma was established on cervicovaginal pap smears in a 62-year-old female presented to gynecological clinic with complaints of stress urinary incontinence. Speculum examination revealed first-degree cervical descent. Smears showed features of squamous cell carcinoma along with fungal spores and fruiting body with hyphae of Aspergillus species. The presence of fruiting bodies and hyphae of Aspergillus species with coexisting squamous cell carcinoma is rare in routine pap smears. True infection needs to be distinguished from contamination by Aspergillus species. Early diagnosis can be established on routine cervicovaginal Pap smear examination.
\end{abstract}

Key Words: Aspergillus, Squamous cell carcinoma, Cervical smear

\section{INTRODUCTION}

Fungal infections of the genitourinary tract are a common cause of itching and vaginal discharge in females. Among the various fungal species, Candida is the most common pathogen seen in cervicovaginal Pap smear whereas infection with other fungi like Paracoccidiodes and Aspergillus are rarely seen $(1,2)$. Aspergillus species is a common fungus causing allergies, severe respiratory infections and invasive aspergillosis. Immunocompromised patients are at increased risk of invasive Aspergillus infection.

Aspergillus species in a cervicovaginal smear is rare. It may represent a contamination or a true infection following prolonged antibiotic therapy or immunosuppression (4). Immunocompromised hosts harboring leukemia or lymphoma and receiving chemotherapy and bone marrow transplant are vulnerable for infection by Aspergillus species.

We report a rare case of squamous cell carcinoma of cervix with concomitant infection with Aspergillus species that was diagnosed on routine cervicovaginal pap smear.

\section{CASE REPORT}

A 62-year-old woman presented to the gynecological clinic with a complaint of stress urinary incontinence. On speculum examination, first-degree cervical descent was noted. The biochemical and hematological investigations were within normal limits. Routine and microscopic urine

(Turk Patoloji Derg 2016, 32:54-56)

Received : 25.10.2012 Accepted : 06.11.2012 examinations were normal. Two slides of cervicovaginal pap smears were sent for cytopathological examination. One slide showed features of squamous cell carcinoma with necrosis and inflammation in the background (Figure 1). The other slide showed mostly superficial and intermediate squamous cells and few atypical squamous cells with high $\mathrm{N}: \mathrm{C}$ ratio and hyperchromatic nucleus along with fungal spores which were seen scattered in the smear along with septate hyphae. The hyphae were seen in association with the fruiting body (Figure 2). The fruiting body was composed of conidiophores and club shaped vesicles covered with a row of phialides. These 2 were capped with long chains of conidiophores. Background showed minimal inflammation. The fungal spores and hyphae with fruiting body were in the same plane as the squamous cells. It was noted that none of the other cervicovaginal pap smears from the gynaecological outpatient clinic showed evidence of fungus thereby excluding the use of contaminated spatulas. The slides were clean and immediate transportation of slides to the cytopathology laboratory was ensured. Thus in this case we ruled out Aspergillus contamination before concluding the cytology report as squamous cell carcinoma and associated infection by Aspergillus species.

The patient was lost to follow up for further investigation and the systemic involvement by the Aspergillus species and especially those of the lungs could not be excluded and the histopathological diagnosis was unavailable.

Correspondence: Prajwala GUPTA

PGIMER, Dr. RML Hospital, Department of Pathology, NEW DELHI, INDIA E-mail: drgoyal4@gmail.com 


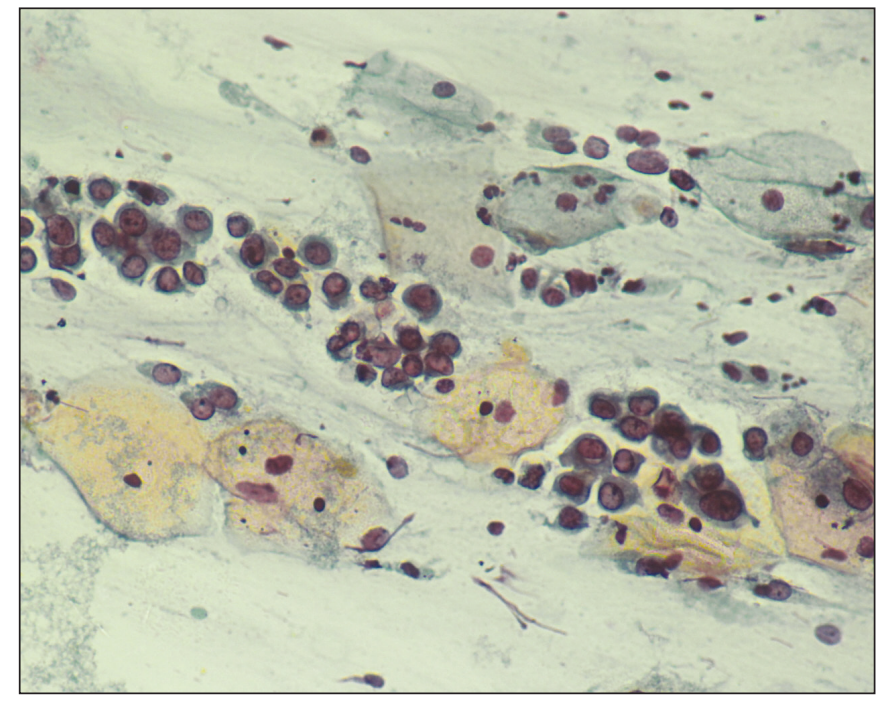

Figure 1: Smear shows malignant squamous cells in a necrotic background (Papanicolaou stain, x400).

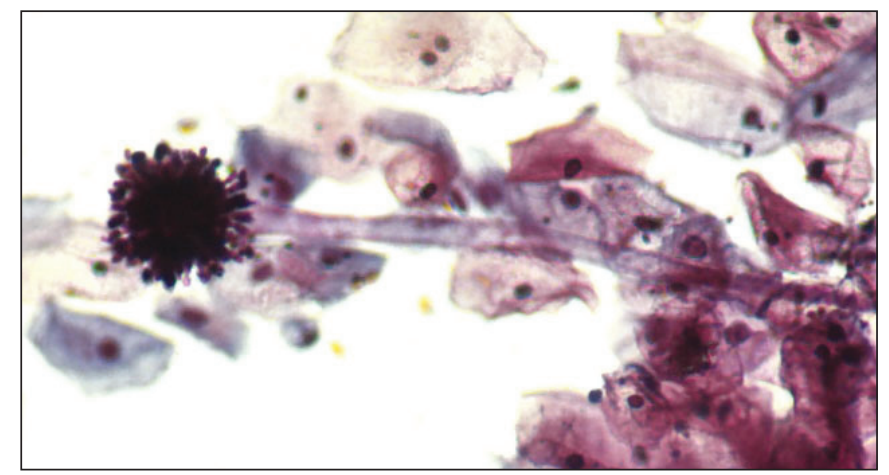

Figure 2: Smear shows the fruiting body of Aspergillus Species (Papanicolaou stain, x400).

\section{DISCUSSION}

The cervicovaginal pap smear is considered an important tool in diagnosing neoplastic lesions in the genital tract. It is also of use in detecting fungal infections especially Candida which shows spores in association with pseudohyphae and budding phenomenon. However, recognition of Aspergillus species requires the presence of characteristic fruiting body with acute angle branching hyphae and spores.

The fruiting body provides clue about the particular fungus and its species $(3,6)$. Determination of the length and width of conidiophores, shape of vesicles, phialides and chains of conidia are noted for the species characterization. In our case the fruiting body resembles Aspergillus species. Several authors emphasize that it is essential to rule out contamination with the Aspergillus species due to use of improperly sterilized spatulas in the colposcopic clinic before diagnosing it as a true infection $(3,5)$. The hyphae lying above the plane of the cervical cells and minimal inflammation suggest that the fungi were not deposited at the same time as the cervical cells thereby favouring contamination of the slide (5). In our case, the fruiting body was characteristic of Aspergillus species and present in the same plane with the cervical cells. Further, it was associated with squamous cell carcinoma that might have resulted in decreased immunity of patient and increased susceptibility for Aspergillus species infection although the systemic involvement could not be ruled out in our case. Only one of the slides showed Aspergillus species with few atypical squamous cells whereas the other slide showed features of squamous cell carcinoma, which rules out the spatula contamination. Aspergillus infection has been reported in a postmenopausal female with features of pelvic inflammatory disease, which resolved following treatment but the patient presented later on with squamous cell carcinoma of the cervix (1).

The infection with Aspergillus species most commonly transmitted by airborne conidia and the lung is the major site of infection. Disseminated disease almost always results from a primary pulmonary infection but it can also occur from skin inoculation or when no likely entry source is identifiable (1). Cytological recognition of the fungus by fluorescence microscopy is possible when it is not easy to distinguish Aspergillus from other filamentous fungi (7).

The identification of Aspergillus species in cervicovaginal smears should prompt the clinician for further investigation to look for systemic focus. The finding of concomitant squamous cell carcinoma in our case suggests the possibility of decreased immunity leading to infection with Aspergillus species. The cervicovaginal pap smears findings and the sterile precautions are helpful in diagnosing a true Aspergillus species infection. Once the factors for the contamination are excluded then the cervicovaginal Pap smear finding of Aspergillus species facilitates an early diagnosis and prompt treatment. This case further concludes the rare finding of the Aspergillus species infection in a patient harbouring squamous cell carcinoma which was diagnosed on cervicovaginal pap smear.

\section{REFERENCES}

1. Deb P, Srivastava A. Aspergillus in a cervico-vaginal smear of an adult postmenopausal female: An unusual case. J Cytol. 2009; 26:123-4.

2. Sullam SA, Mahfouz AA, Dabbous NI, el-Barrawy M, el- Said MM. Reproductive tract infections among married women in upper Egypt. East Mediterr Health J. 2001;7:139-46. 
3. Chandra S, Gaur D, Meena H, Chaturvedi J, Kishore S. An unusual presentation of aspergillus species in a routine cervicovaginal pap smear. Acta Cytol. 2009; 53:229-31.

4. Hoda RS, Colello C, Roddy M, Houser PM. "Fruiting body" of aspergillus species in a routine cervico-vaginal smear (Pap test). Diag Cytopathol. 2005;33:244-5.

5. Riben M, Katz RL. Fungal fruiting bodies in a pap smear: Contamination or infection? Acta Cytol. 2006;50:112-4.
6. Martinez- Giron R, Fernandez- Garcia C. Aspergillus/penicillium sp. Spores as a contaminant on conventional pap smear. Diag Cytopathol. 2009;37:899-900.

7. Hettlich C, Kupper TH, Wehle K, Pfitzer P. Aspergillus in the papanicolaou stain: Morphology, fluorescence and diagnostic feasibility. Cytopathology. 1998;9:381-8. 